\title{
Análisis estadístico de choques viales a nivel distrital para la gran área metropolitana
}

\author{
Statistical analysis of road crashes at the district level for the greater metropolitan area
}

\section{Darío Vargas Aguilar}

Programa de investigación en Desarrollo Urbano Sostenible Universidad de Costa Rica

Costa Rica

dario.vargasaguilar@ucr.ac.cr

\section{Jonathan Agüero Valverde, Ph.D.}

Programa de investigación en Desarrollo Urbano Sostenible

Universidad de Costa Rica

Costa Rica

jonathan.aguero@ucr.ac.cr

Fecha de recepción: 01 de julio de 2020 / Fecha de aprobación: 23 de noviembre de 2020

\section{RESUMEN}

Debido al incremento constante de choques viales que ocurre en el país, se buscó determinar las características socioeconómicas de la población y las variables de uso de suelo relacionadas con la frecuencia con la que estos ocurren a nivel de distrito en la Gran Área Metropolitana (GAM), de manera que fuera posible incluir criterios de seguridad vial en las políticas de planificación urbana.

Se elaboraron modelos estadísticos de estimación de choques, utilizando la distribución binomial negativa a partir del uso de suelo de la GAM. Se incluyeron las variables socioeconómicas de la población y variables asociadas a la red vial. Se determinaron las variables representativas en cada uno de los tipos de choque analizados como, por ejemplo, la población total, y se realizó la estimación de choques esperados para cada uno de los distritos en estudio. Adicionalmente, se determinaron los distritos con mayor exceso de frecuencia, donde sobresale el distrito de Alajuela que resultó ser el más problemático. También resultaron con importantes excesos de choques los distritos de Uruca, Hospital, San Nicolás, Río Segundo, Heredia, entre otros.

Palabras clave: Choques viales; modelos estadísticos; seguridad vial; exceso de choques.

\section{ABSTRACT}

Due to the constant increase in the number of road crashes that occur in the country, it was sought to determine the socio-economic characteristics of the population and the variables of land use related to the frequency of road crashes at the district level in the Greater Metropolitan Area (GAM). This will make possible to include road safety criteria in urban planning policies.

Statistical models of crash estimation were developed, using the negative binomial distribution based on the land use of the GAM. The socioeconomic variables of the population and variables associated with the road network were included. The representative variables in each of the types of crashes analyzed were determined, such as the total population, and the estimation of expected crashes was performed for each of the districts under study. Additionally, the districts with the highest frequency were determined, where the Alajuela district stands out, which proved to be the most problematic. The districts of Heredia, Uruca, Hospital, San Nicolás, Río Segundo, among others, also resulted in significant excesses of crashes.

Keywords: Road crashes; statistical models; road safety; excesses of crashes. 


\section{INTRODUCCIÓN}

La seguridad vial es un área de estudio que cada año adquiere más importancia, ya que el crecimiento en el nivel de motorización mundial ha agravado la problemática de los choques viales, que han alcanzado el punto de ser declarado un problema de salud pública por parte de la Organización Mundial de la Salud y formar parte de la agenda para el desarrollo sostenible por parte de las Naciones Unidas. Esto genera anualmente la perdida de millones de vidas humanas, así como serias lesiones a otros millones de personas (World Health Organization [WHO], 2018). Adicionalmente, a estas pérdidas humanas deben añadirse los costos económicos asociados a las demoras y daños a la propiedad (ProDUS, 2015). Es por esto por lo que surge la necesidad de buscar maneras de reducir la ocurrencia y gravedad de los choques viales.

A nivel internacional existen investigaciones centradas en relacionar las características del uso del suelo con la frecuencia de choques viales, por ejemplo, en 2013 Srinivas Pulugurtha; Venkata Rammana Duddu y Yashaswi Kotagiri realizaron la investigación "Traffic analysis zone level crash estimation models based on land use". Utilizando datos de choques viales y uso de suelo del año 2005, el estudio analizó las denominadas zonas de análisis de tráfico en la ciudad de Charlotte, en el condado de Mercklenburg en Carolina del Norte, relacionándolas con diferentes categorías de uso de suelo como, por ejemplo, residencial, mixto, comercial e industrial. Los investigadores utilizaron un modelo binomial negativo y verificaron los resultados obtenidos mediante una prueba chi-cuadrada $\left(\chi^{2}\right)$ con $95 \%$ de confianza. Determinaron que los diferentes usos de suelo presentaban una estrecha relación positiva con la ocurrencia de choques viales.

Otro ejemplo es la investigación de 2016 titulada "Investigating the Effects of Traffic, Socio-Economic and Land Use Characteristics on Pedestrian and Bicycle Crashes: A Case Study of Melbourne, Australia", Richard Amoh-Gyimah utilizó variables socioeconómicas, densidad poblacional y de uso de suelo para generar modelos estadísticos que permitieran determinar la relación de estas variables con los atropellos y choques a ciclistas. Para los modelos fue utilizada la distribución binomial negativa y se determinó que la cantidad de kilómetros viajados por vehículo, la cantidad de adultos mayores y el porcentaje de hogares sin automóvil, tienen una correlación positiva con respecto a la cantidad de atropellos a ciclistas y peatones. A nivel latinoamericano las investigaciones de este tipo son escasas. En el caso de Costa Rica no existen investigaciones en el tema, por lo que es necesario incursionar en este campo.

La presente investigación tiene como objetivo determinar las variables relacionadas con la frecuencia de choques viales en los distritos de la Gran Área Metropolitana de Costa Rica (GAM). Para ello fue requerido elaborar modelos estadísticos a partir de características socioeconómicas y de uso del suelo que permitieran identificar las variables que inciden en la ocurrencia de choques viales. Se considera que, al identificar estas variables, será posible incluir aspectos relacionados al uso del suelo en las políticas y normativas de seguridad vial. Esto permitiría mejorar la planificación territorial.

\section{METODOLOGÍA}

\section{Bases de datos}

Se utilizó información de diferentes bases de datos tales como el uso de suelo escala 1:1000 del proyecto de Planificación Regional y Urbana de la GAM (PRUGAM) (Instituto Geográfico Nacional, 2008), proyecciones de población (Instituto Nacional de Estadística y Censos de Costa Rica, 2014), información socioeconómica obtenida del censo poblacional 2011 (Instituto Nacional de Estadística y Censos de Costa Rica, 2011), inventario de carreteras del Ministerio de Obras Públicas y Transportes (2012) y bases de datos de choques viales del Consejo Nacional de Seguridad Vial (COSEVI) (Consejo de Seguridad Vial, 2016).

Se creó una base de datos unificada, para ello fue necesario como trabajo previo completar el uso de suelo del PRUGAM en el área de estudio, para lo cual se utilizaron fotografías aéreas y sistemas de información geográfica. El uso de sistemas de información geográfica también fue requerido en otras bases de datos, esto con el fin de relacionar las diferentes variables con su respectivo distrito. Una vez unificadas las bases de datos se determinó las posibles variables de interés para incluir en los modelos, estas variables fueron seleccionadas con base en revisión bibliográfica. Se determinó además las estadísticas descriptivas de estas variables, algunas de las cuales se muestran en el Cuadro 1. 
Cuadro 1. Estadísticas descriptivas de algunas variables analizadas

\begin{tabular}{|c|c|c|c|c|c|}
\hline Variable & Máximo & Mínimo & Promedio & $\begin{array}{l}\text { Desviación } \\
\text { estándar }\end{array}$ & Mediana \\
\hline Uso de suelo tipo bosque y protección & $54,8 \%$ & $0,0 \%$ & $16,6 \%$ & $14,3 \%$ & $12,2 \%$ \\
\hline Uso de suelo tipo comercial y servicios & $33,5 \%$ & $0,0 \%$ & $3,9 \%$ & $6,1 \%$ & $1,4 \%$ \\
\hline Uso de suelo tipo industrial & $29,2 \%$ & $0,0 \%$ & $4,0 \%$ & $5,9 \%$ & $1,8 \%$ \\
\hline Uso de suelo tipo agropecuario & $93,6 \%$ & $0,6 \%$ & $41,8 \%$ & $23,1 \%$ & $44,2 \%$ \\
\hline Uso de suelo tipo residencial & $93,6 \%$ & $1,4 \%$ & $33,7 \%$ & $23,7 \%$ & $30,4 \%$ \\
\hline Población entre los 0 y los 14 años & $29,8 \%$ & $9,4 \%$ & $22,6 \%$ & $3,4 \%$ & $23,0 \%$ \\
\hline Población entre los 15 y los 24 años & $21,2 \%$ & $14,3 \%$ & $18,0 \%$ & $1,4 \%$ & $17,8 \%$ \\
\hline Población entre los 25 y los 64 años & $65,6 \%$ & $42,4 \%$ & $52,4 \%$ & $3,7 \%$ & $52,4 \%$ \\
\hline Población de 65 años o más & $10,8 \%$ & $4,6 \%$ & $7,0 \%$ & $1,3 \%$ & $6,6 \%$ \\
\hline Población total en miles de habitantes & 79,41 & 0,90 & 14,45 & 11,75 & 10,4 \\
\hline Vehículo Kilómetro año $2012^{*}$ & 0,68 & 0,00 & 0,09 & 0,12 & 0,05 \\
\hline Vehículo Kilómetro año 2013* & 0,68 & 0,00 & 0,09 & 0,12 & 0,05 \\
\hline Vehículo Kilómetro año 2014* & 0,71 & 0,00 & 0,10 & 0,12 & 0,05 \\
\hline
\end{tabular}

*Vehículos Kilómetro en millones

\section{Elaboración del Modelo Binomial Negativo}

Para elaborar modelos estadísticos, se utilizó la distribución binomial negativa. Se consideró que el logaritmo natural del número estimado de choques es una combinación lineal de variables independientes. Asimismo, que la distribución binomial negativa puede tratarse como una distribución de Poisson con promedio $\lambda$, donde $\lambda$ es una variable aleatoria con distribución Gamma (Agüero-Valverde, 2016). Entonces, para una variable discreta aleatoria, $Y$, y la frecuencia observada i donde $Y i=1, \ldots$, , el modelo binomial negativo esta dado por la ecuación 1.

$$
\ln \lambda=\beta_{i} x_{i}+\varepsilon_{i}
$$

Para este modelo $\lambda$ es la cantidad esperada de eventos por distrito, $x_{i}$ es un vector de variables y $\varepsilon$ representa el error de especificación o la heterogeneidad entre secciones. Donde $\beta i$ es el cambio proporcional en el número esperado de eventos por período de una unidad de cambio en una variable independiente $x_{j}$ (Fuentes y Hernández, 2009).

Para la elaboración de los modelos se utilizó el software estadístico $\mathrm{R}$, se consideró un modelo base que relacionó de manera proporcional la cantidad total de choques ocurridos por distrito y el logaritmo natural de los vehículo-kilómetro en el mismo distrito. El modelo base fue modificado, agregando o eliminado variables, hasta obtener un modelo capaz de realizar una mejor aproximación de los datos que el modelo previo. Para determinar si un modelo realiza una mejor aproximación que el modelo base, es necesario verificar la bondad de ajuste. Para ello, se utilizaron indicadores estadísticos como la seudo- $\mathrm{R}^{2}$ de McFadden o la prueba de relación del logaritmo de verosimilitud.

En el caso de la seudo- $\mathrm{R}^{2}$ de McFadden, se tiene que el modelo presenta un mejor ajuste respecto a los datos observados cuando el valor de $\mathrm{R}^{2}$ se acerca a uno y disminuye cuando se aleja de este valor, siempre en el intervalo $[0,1]$. La prueba de relación del logaritmo de verosimilitud establece el aporte de una determinada variable al ajuste de los datos respecto al modelo base existente, de manera que es posible determinar la independencia de una variable respecto a las presentes en el modelo. Para efectos de esta prueba se utilizó un grado de confianza de 0,05; por lo tanto, se consideró que un modelo presenta mejor ajuste si $P_{x}^{2}(D, A)<0,05$, donde $D$ representa el doble del logaritmo de la diferencia de verosimilitud entre el modelo base y el modelo anidado; y $A$ representa la diferencia entre los grados de libertad del modelo base y el modelo anidado.

También se utilizó el criterio de Akaike Information Criterion (AIC, por sus siglas en inglés) para realizar comparaciones entre modelos no anidados, según Caballero (2011), el AIC permite identificar el mejor modelo entre varias opciones, ya que se puede interpretar como una aproximación de la bondad de ajuste del modelo. Este criterio cuenta con dos partes, una que premia la bondad de ajuste del modelo, mientras la segunda parte penaliza la inclusión de parámetros adicionales según el principio de parsimonia, de manera que el modelo con menor valor de AIC es el mejor de los considerados. 


\section{Determinación del exceso de frecuencia de choques}

Una vez determinadas las variables de cada modelo y sus respectivos coeficientes, se estimó la cantidad de choques para cada distrito mediante la función de desempeño asociada al respectivo modelo. Posteriormente, con el fin de determinar los excesos de frecuencia, se calculó un valor de ajuste por peso. Dicho ajuste contempla el parámetro de sobre dispersión, por lo que permite determinar la confiabilidad de la función de desempeño, de manera que, al disminuir la dispersión, la función de desempeño es más confiable (Agüero-Valverde, 2016). Este factor de peso se calcula según la ecuación 2.

$$
w=\frac{1}{1-\emptyset\left(N_{\text {estimado }}\right)}
$$

Donde $\varnothing$ es el parámetro de sobre dispersión y w se conoce como peso de Bayes empírico. Una vez determinado el peso de Bayes empírico es posible determinar la cantidad de choques esperados en un distrito mediante el uso de la ecuación 3.

$$
N_{\text {esperado }}=w N_{\text {estimado }}+(1-w) N_{\text {observado }}
$$

Conociendo el valor de choques esperados se determina el exceso de frecuencia de choques por distrito según la ecuación 4 .

$$
\Delta=N_{\text {esperado }}-N_{\text {estimado }}
$$

\section{RESULTADOS}

\section{Modelos estadísticos}

Para la elaboración de los modelos estadísticos se partió de una hipótesis nula, donde la cantidad de choques esperados en cada distrito era igual a la media de la distribución de datos. Posteriormente, se determinó una variable inicial con el fin de generar un modelo diferente del modelo nulo. Dicha variable fue el logaritmo natural del vehículo-kilómetro.

Se realizó una prueba de bondad de ajuste con el fin de determinar si la inclusión de esta variable al modelo genera una mejora en la predicción de los datos observados. Como criterio para determinar la bondad de ajuste se utilizó la prueba de relación del logaritmo de verosimilitud a un nivel de confianza de 0,05 . Una vez obtenido el nuevo modelo nulo, este se utilizó como base para la generación de modelos más complejos. En los casos en los que existieron dos o más modelos cuya relación del logaritmo de verosimilitud fuera de 0,05 o menor, se utilizó el criterio de AIC y el pseudo $\mathrm{R}^{2}$ para determinar el modelo con mejor ajuste. Se continuó realizando el procedimiento de forma iterativa hasta que ningún nuevo modelo tuviera un valor de 0,05 o menor en la prueba de bondad de ajuste.

Mediante el procedimiento anterior, para el modelo de estimación de choques totales, se obtuvieron los parámetros mostrados en el Cuadro 2. A su vez, para el modelo de estimación de atropellos a personas, se obtuvieron los

\begin{tabular}{|c|c|c|c|c|}
\hline Variable & $\begin{array}{l}\text { Promedio de } \\
\text { coeficiente }\end{array}$ & $\begin{array}{l}\text { Error estándar de } \\
\text { coeficiente }\end{array}$ & t-Student & $P(>|t|)$ \\
\hline Intercepción & 1,630 & 0,255 & 6,4 & $1,70 \mathrm{e}-10$ \\
\hline Log (Población total) & 0,944 & 0,040 & 23,4 & $<2 \mathrm{e}-16$ \\
\hline Log (Vehículo-kilómetro) & 0,254 & 0,021 & 12,1 & $<2 \mathrm{e}-16$ \\
\hline $\begin{array}{l}\text { Porcentaje de territorio dedicado a uso de suelo } \\
\text { comercial y de servicios }\end{array}$ & 8,765 & 0,605 & 14,5 & $<2 \mathrm{e}-16$ \\
\hline Porcentaje de personas de 65 años o más & 19,266 & 2,879 & 6,7 & $2,22 \mathrm{e}-11$ \\
\hline
\end{tabular}
parámetros mostrados en el Cuadro 3.

Cuadro 2. Modelo de estimación de cantidad de choques totales anuales por distrito para la GAM

Nota: Desviación nula: 2373,15. Desviación residual: 509,43. Theta: 2,438. Error estándar: 0,155. 2xLog(Verosimilitud): -5421,976. Pseudo R²: 0,785. AIC: 5433,8. Desviación nula solo con constante: 590,24. $P_{x}^{2}(\mathrm{z}): 1,11 \mathrm{e}-16$ 
Cuadro 3. Modelo de estimación de atropellos a personas por distrito para la GAM

\begin{tabular}{|c|c|c|c|c|}
\hline Variable & $\begin{array}{c}\text { Promediode } \\
\text { coeficiente }\end{array}$ & $\begin{array}{c}\text { Error estándar de } \\
\text { coeficiente }\end{array}$ & t-Student \\
\hline Intercepción & 2,140 & 0,345 & 6,2 & $5,57 \mathrm{e}-10$ \\
\hline Log (Población total) & $1,054)$ & 0,058 & 18,2 & $<2 \mathrm{e}-16$ \\
\hline Log (Vehículos kilómetro) & 0,085 & 0,028 & 3,0 & 0,00247 \\
\hline Porcentaje de territorio dedicado a uso de suelo residencial & 0,057 & 0,217 & 2,3 & 0,01936 \\
\hline Porcentaje de personas entre los 0 y los 14 años & $-13,398$ & 1,543 & $-8,7$ & $<2 \mathrm{e}-16$ \\
\hline
\end{tabular}

Nota: Desviación nula: 1495,00. Desviación residual: 493,47. Theta: 2,645. Error estándar: 0,246. 2xLog(Verosimilitud): -2560097. Pseudo $R^{2}$ : 0,67. AIC: 2572,1. Desviación nula solo con constante: 545,69. $P_{x}^{2}(\mathrm{z}): 1,24 \mathrm{e}-10$

También es posible expresar estos modelos en forma algebraica como se muestra en la ecuación 5 y la ecuación 6 .

- Estimación de choques totales anuales por distrito

$$
C_{T}=X^{0,944} Y^{0,254} e^{1,63+8,76 Z+19,264 W}
$$

- Estimación de choques de tipo atropello a persona por año y por distrito

$$
C_{P}=X^{1,05} Y^{0,085} e^{2,14+0,057 T-13,40 U}
$$

Donde $C_{T}$ es la cantidad de choques totales esperados durante un año en un distrito, $C_{P}$ es la cantidad de choques de tipo atropello a persona esperados durante un año en un distrito, $X$ es la población total en miles de habitantes, $Y$ es la cantidad de vehículo-kilómetro de las rutas nacionales en millones de vehículos, $T$ es la fracción del distrito destinada a uso de suelo residencial, $U$ es la fracción de la población entre 0 y 14 años, $W$ es la fracción de la población con 65 años o más y $Z$ es la fracción del distrito destinada a uso de suelo comercial y de servicios.

De los modelos anteriores, se puede apreciar en el caso del modelo de estimación de choques totales mostrado en el Cuadro 1, que la variable de mayor peso es el porcentaje de personas adultos mayores en el distrito. Según el modelo obtenido un incremento del $1 \%$ en la población adulta mayor del distrito representa un incremento del $21 \%$ en la cantidad de choques estimados. En el caso del porcentaje del territorio destinado al uso de suelo comercial y de servicios el modelo refleja que un incremento del $1 \%$ en la variable genera un aumento del $9 \%$ en la cantidad de choques estimados.

Para la población total del distrito, se obtuvo que al aumentar la cantidad de habitantes del distrito se aumenta la cantidad de choques estimados mediante el modelo. Dichos incrementos en la cantidad de choques son menores con cada mil habitantes adicionales, ya que se presenta una tasa decreciente de aumento. Este mismo efecto sucede con la variable vehículo-kilómetro, la cual también presenta una tasa decreciente de aumento. Por otro lado, al comparar los valores de los exponentes de estas dos variables, se determinó que se generan mayores incrementos en los choques estimados al aumentar la población que al aumentar el valor de vehículo-kilómetro.

Para el modelo de estimación de choques de tipo atropello a persona, se obtuvo que la variable población total del distrito presenta un aporte positivo al modelo. Esta variable presenta una tasa de aporte creciente, lo que implica que al crecer la población del distrito aumentará la cantidad de atropellos estimados y a su vez, cada unidad (mil habitantes) adicional generará un mayor incremento en la cantidad de atropellos que la unidad previa. En este modelo cobra relevancia el uso de suelo residencial, para esta variable se espera que un incremento de $1 \%$ genera un aumento del $0,01 \%$ en la cantidad de atropellos a personas estimado. Otra variable importante es el porcentaje de la población que se encuentra entre los 0 a los 14 años, esta variable genera un aporte negativo por lo que por cada incremento de $1 \%$ representa una disminución del $14,3 \%$ de los atropellos estimados.

Las variables que fueron identificadas como relevantes en ambos modelos son consistentes con las investigaciones mencionadas en la sección de introducción, así como otras relacionadas al tema, por ejemplo: "Predicting Local Road Crashes Using Socio-economic and Land Cover Data" elaborada por Kai Wang, John Ivan, Amy Burnicki y Sha Mamun (2017).

Mediante los modelos anteriores se realizó una estimación de los valores esperados para cada uno de los años de los que se tiene registro. Para esto, se utilizaron las ecuaciones 5 y 6 en conjunto con los datos de uso de suelo, tránsito y socioeconómicos respectivos de cada distrito. Debido a que el área de estudio 
comprende un total de 160 distritos, únicamente se presenta la información para los 16 distritos con mayor cantidad de choques estimados. En la Figura 1 y la Figura 2 se muestra la cantidad estimada de choques totales y la cantidad de choques de tipo atropello a persona, respectivamente.

En la Figura 1 es posible apreciar que, con excepción de Alajuela, los distritos con mayor cantidad de choques totales estimados se encuentran ubicados en la provincia de San José, mayoritariamente en el cantón del mismo nombre. El distrito de Pavas es el que presenta más choques estimados superando los 4300 choques para cada uno de los años en estudio, mientras que el distrito de Alajuela se ubica en la posición 16 y la cantidad de choques estimada oscila entre 900 y 1000 choques anuales. Sin embargo, según los datos suministrados por el COSEVI el distrito de Alajuela es el que presenta mayor cantidad de choques cada año, esta diferencia entre los choques ocurridos y los estimados implica que existe un exceso de frecuencia de choques para ciertos distritos como es el caso de Alajuela.

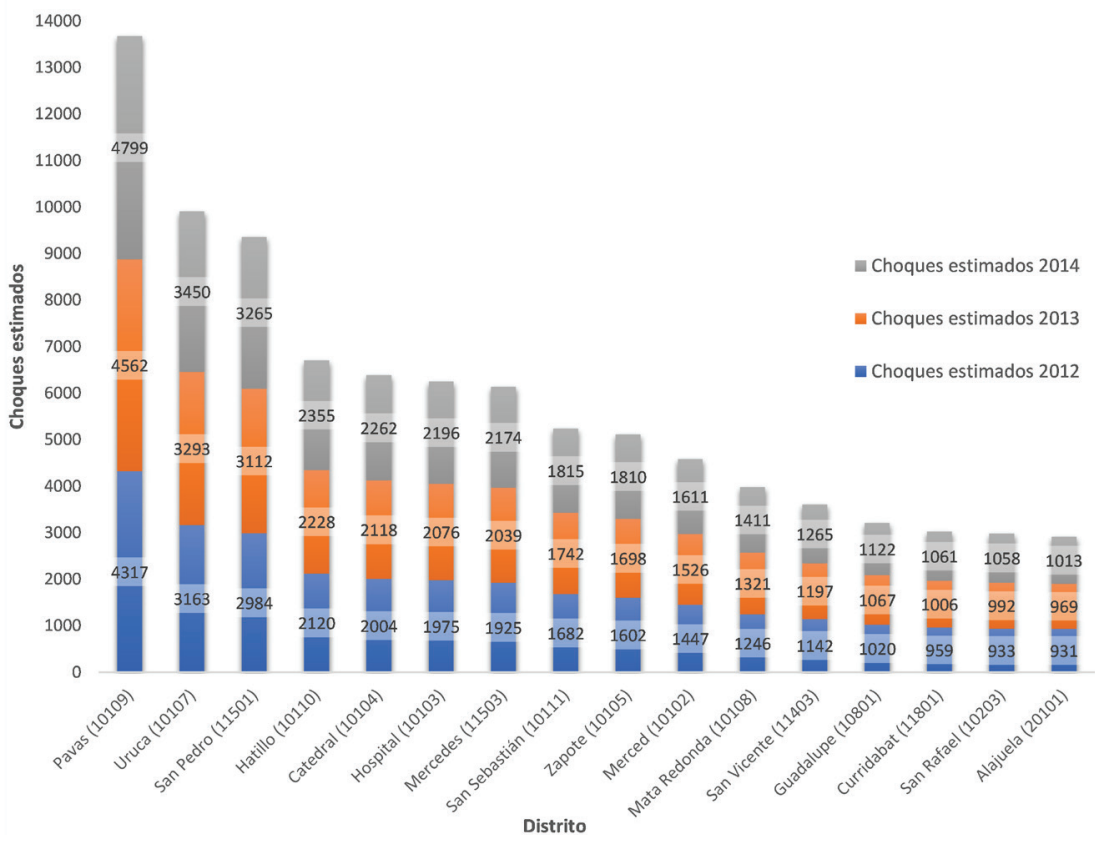

Figura 1. Cantidad total de choques viales estimados según distrito

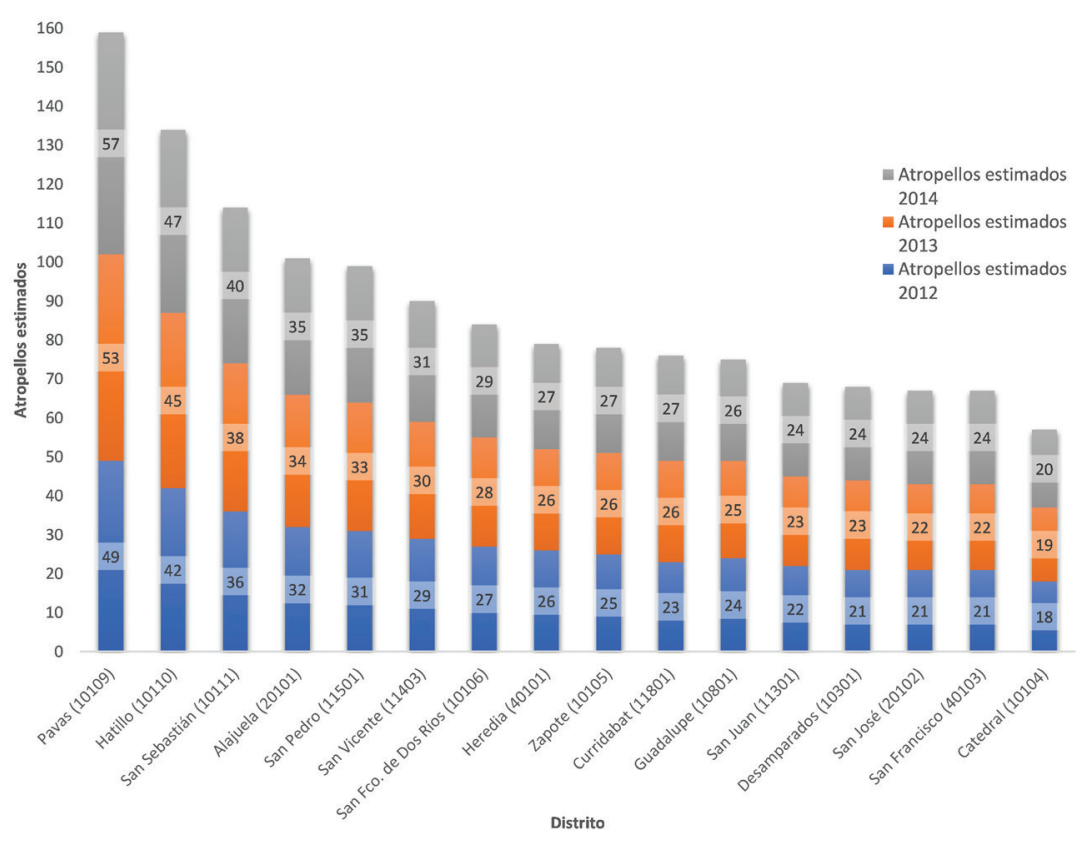

Figura 2. Cantidad estimada de atropellos a personas según distrito 
Una situación similar ocurre con los choques de tipo atropello a persona, en la Figura 2 se muestra que los distritos con mayor cantidad de atropellos se encuentran en las cercanías de importantes ciudades como San José, Heredia y Alajuela, siendo distritos con vocación urbana. Para este caso nuevamente el distrito de Pavas presenta la mayor cantidad estimada de choques, no obstante, esto difiere de la realidad según los datos suministrados por el COSEVI.

\section{Exceso de frecuencia}

Con el fin de determinar cuáles de los distritos analizados presenta mayor problema de choques se calculó el exceso de frecuencia para cada caso, esto se realizó siguiendo el procedimiento descrito en la sección referente a metodología. Se determinó que, para el caso de choques totales, 53 de los 160 distritos en estudio presentan exceso de frecuencia, siendo el distrito de Alajuela el que más exceso presenta con valores anuales superiores a mil choques adicionales. En la Figura 3 se muestran los distritos con más exceso de frecuencia, además de Alajuela, se detectó que Desamparados, Heredia, Ulloa, Curridabat y San Rafael de Escazú no solo presentan un elevado exceso de frecuencia, sino que forman parte de los distritos con más choques anuales reportados por el COSEVI.

Por otro lado, al analizar la distribución espacial de los distritos mostrados en la Figura 3, se puede detectar que existen regiones donde se concentra el exceso de frecuencia, para los choques totales se detectaron tres grupos importantes, el primero formado por Alajuela, Río Segundo y San Joaquín, el segundo por Pozos, Santa Ana, Escazú y San Rafael de Escazú y el tercer grupo formado por Oriental, Occidental y San Nicolas de Cartago, como se observa en la Figura 4. Debe señalarse que estos tres grupos de distritos son atravesados por importantes rutas primarias como es el caso de las rutas nacionales 2, 3 y 27.

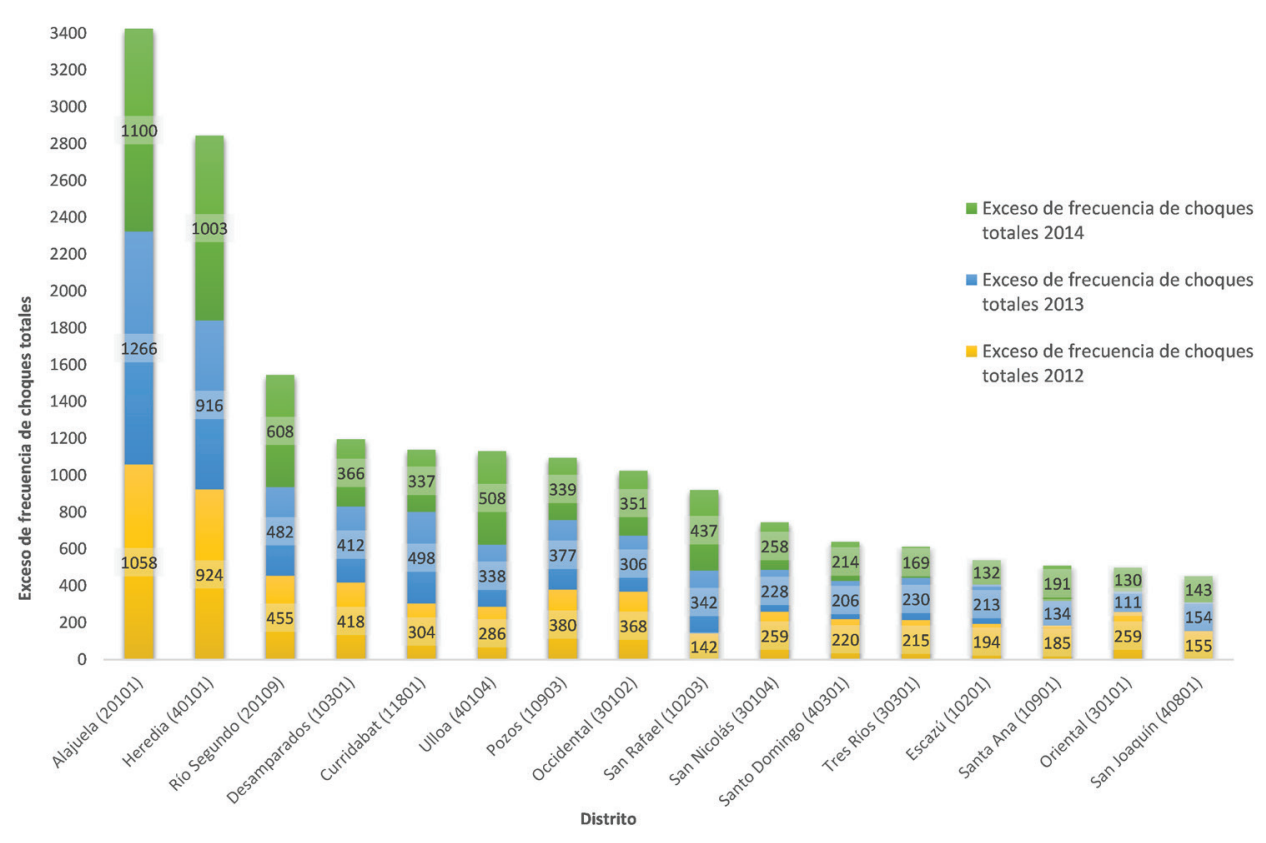

Figura 3. Exceso de choques totales para los distritos con mayor exceso de frecuencia 


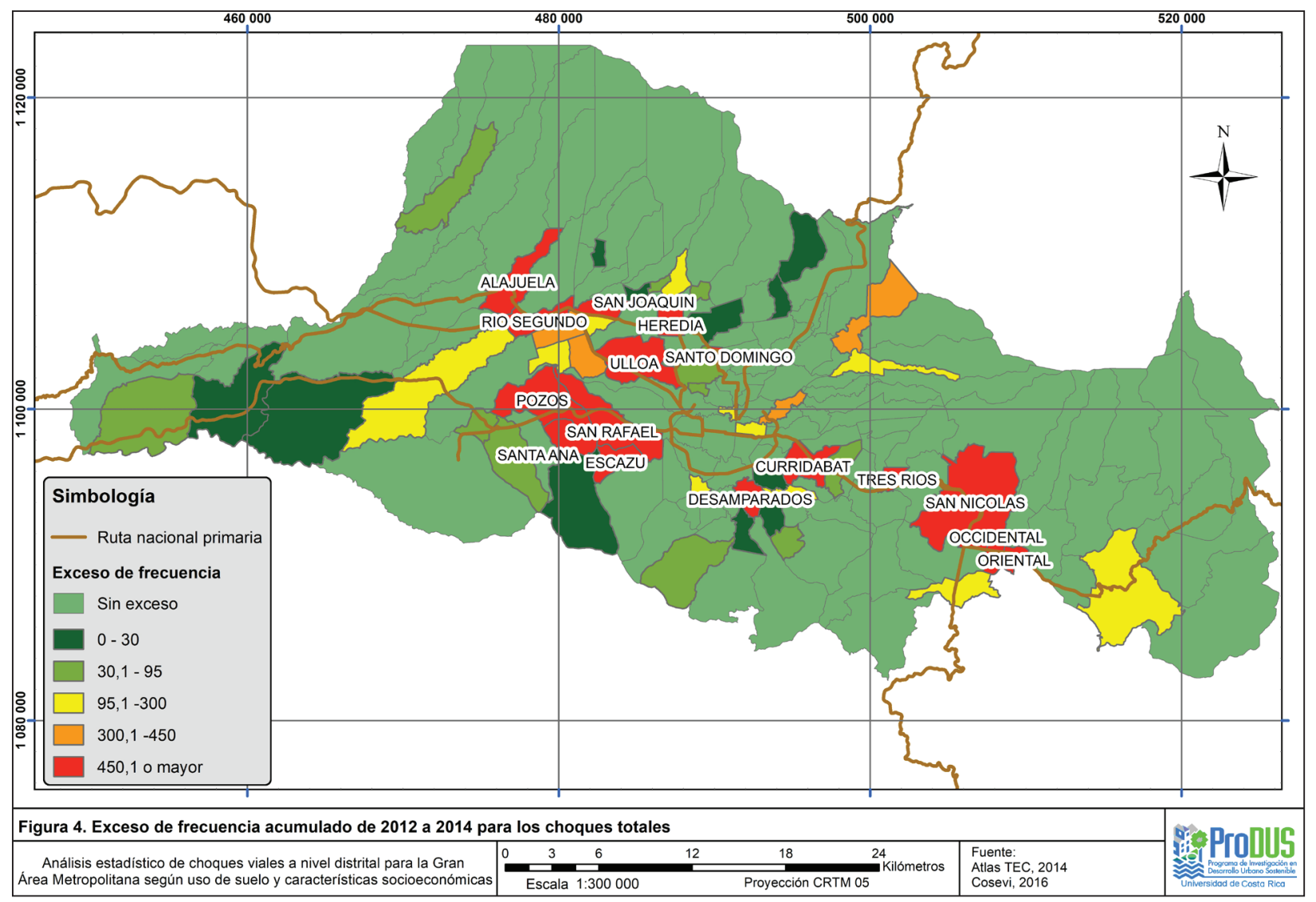

Figura 4. Distribución espacial de los distritos con exceso de choques totales

En el caso de los choques de tipo atropello a persona, la Figura 5 se muestra los distritos con más exceso de frecuencia. Se aprecia que el año 2013 fue especialmente complicado para este tipo de choques ya que se da un incremento importante respecto a la cantidad del año 2012, esto ocurre en distritos como Alajuela, Hospital, Merced, Desamparados, Carmen y Guadalupe. Asimismo, se da un fenómeno similar de incremento durante el año 2014 para distritos como Heredia, Ulloa y San Rafael de Escazú. También se puede observar que existen al menos siete distritos donde el exceso de frecuencia es de 15 o más atropellos anuales. En estos distritos el exceso de frecuencia oscila entre el $40 \%$ y el $80 \%$ de la cantidad de

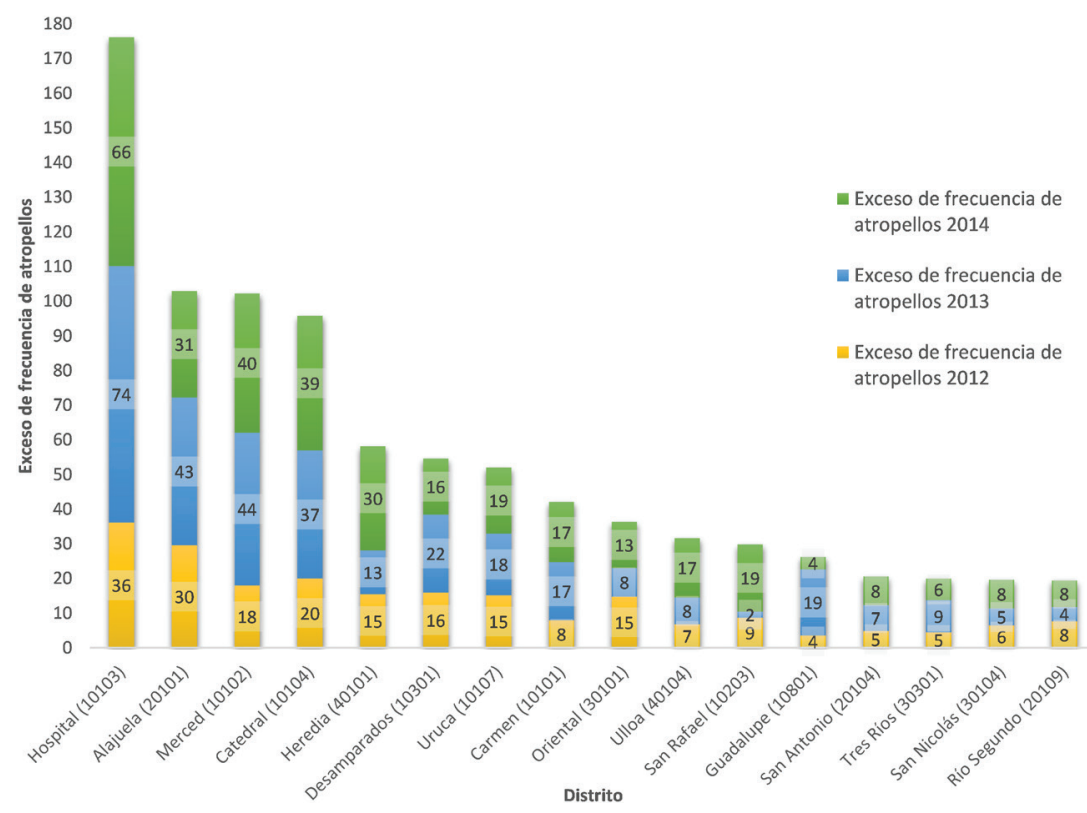

Figura 5. Exceso de atropellos para los distritos con mayor exceso de frecuencia 
choques esperados para su correspondiente distrito. Estos valores especialmente alarmantes al considerar que los peatones son los usuarios más vulnerables de la red vial.

En el caso de los atropellos, también es posible detectar bloques en los que se concentra el exceso de frecuencia, existe un área importante formada por los distritos de Ulloa, Uruca, Guadalupe, Catedral, Carmen, Hospital y Merced, siendo los tres últimos los que reportan más exceso de atropellos superando los 96 anuales en cada distrito. Adicionalmente, existe una pequeña región formada por los distritos de Alajuela y San Antonio, como se muestra en el Figura 6. Se debe señalar que los distritos con más exceso de frecuencia de atropellos abarcan los distritos que forman las principales ciudades del país, algunas de estas ciudades, como es el caso de San José y Cartago, están formadas por más de un distrito.

\section{CONCLUSIONES}

Se determinó que la población total del distrito es una variable representativa en todos los modelos evaluados. Esta variable tiene un impacto directo y positivo en la incidencia de choques viales, de manera que incrementos en la población de un distrito repercuten en una mayor frecuencia de choques. Misma situación ocurre con la variable vehículo-kilómetro.

El porcentaje de uso de suelo comercial y de servicios es una variable representativa para el modelo de choques totales, la variable presenta un aporte positivo al modelo.

El porcentaje de uso de suelo residencial es una variable representativa en la estimación de atropellos a personas, se determinó que, a mayor porcentaje de uso de suelo residencial, mayor la cantidad de atropellos en el distrito.

Se determinó que el porcentaje de la población con edad igual o mayor a 65 años es una variable representativa, que aporta de manera positiva a la cantidad de choques totales estimados por año en un determinado distrito. Esta variable puede tener un efecto en el modelo por cuanto las personas en este rango edad presentan menos velocidad de reacción, más problemas de visión y movilidad que podrían afectar sus habilidades como conductores y, por consiguiente, verse involucrados en choques.

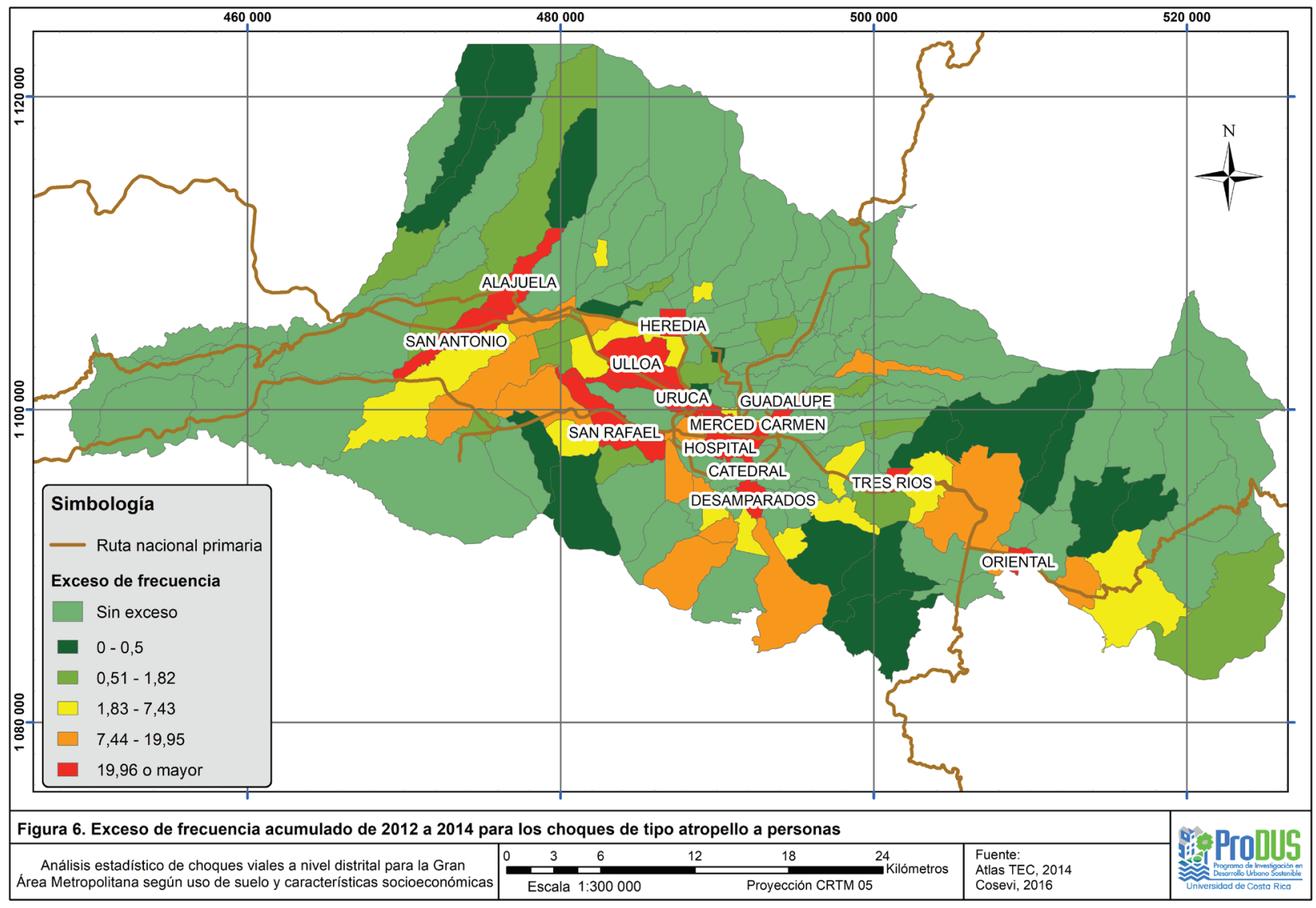

Figura 6. Distribución espacial de los distritos con exceso de atropellos 
Se determinó que el porcentaje de habitantes entre las edades de 0 a 14 años es una variable representativa y que influye de manera negativa en la incidencia de atropellos en un distrito, por ende, entre mayor la población entre los 0 a los 14 años, se estima una menor cantidad de atropellos a personas. Esta variable puede tener efecto en el modelo, por cuanto en las zonas con más niños y adolescentes usualmente los conductores tienden a estar más alerta ante posibles situaciones imprevistas. Por otro lado, existen más zonas de velocidad restringida como escuelas y colegios lo que genera que los conductores viajen a menor velocidad, mejorando su posibilidad de reacción.
Se determinó la existencia de bloques de distritos con exceso de choques para todas las categorías de choques analizadas, mostrando la posibilidad de realizar análisis espaciales de concentración de choques con una unidad geoespacial diferente del distrito, con el fin de determinar los motivos de estos excesos de frecuencia a nivel regional.

El distrito de Alajuela es el distrito más problemático en términos de choques viales, por lo que requiere de especial atención por parte de las autoridades competentes.

\section{REFERENCIAS}

Agüero-Valverde, J. (2016). Determinación de rutas con potencial de mejora utilizando funciones de desempeño de seguridad vial: caso de Costa Rica. Infraestructura Vial, 18(32), 39-52. DOI: 10.15517/iv.v18i32.29783

Amoh-Gyimah, R., Sarvi, M. y Saberi, M. (2016). Investigating the effects of traffic, socioeconomic, and land use characteristics on pedestrian and bicycle crashes: a case study of Melbourne, Australia (No. 16-1931). En Transportation Research Board 95th Annual Meeting, Washington DC, United States.

Caballero, F. (2011). Selección de modelos mediante criterios de información en análisis factorial. Aspectos teóricos y computacionales. (Tesis doctoral, Universidad de Granada). Recuperado de: https://hera.ugr.es/tesisugr/19964808.pdf

Consejo de Seguridad Vial (2016). Datos de accidentes de Tránsito en la Gran Área Metropolitana periodo 2012-2014 [Fichero de datos sin publicar]. San José, Costa Rica.

Fuentes, C. M. y Hernández, V. (2009). La estructura espacial urbana y la incidencia de accidentes de tránsito en Tijuana, Baja California (2003-2004). Frontera norte, 21(42), 109-138.

Instituto Geográfico Nacional (2008). Hojas cartográficas de Uso de la Tierra en la Gran Área Metropolitana del Proyecto PRUGAM (1:10,000), San José Costa Rica. Recuperado de: https://www.mivah.go.cr/PRUGAM_Cartografia_Alfabetica.shtml

Instituto Nacional de Estadística y Censos de Costa Rica (2011). Censo 2011 Distribución porcentual de los hogares por número de carencias críticas, según distrito [Fichero de datos]. Obtenido de http://www.inec.go.cr/pobreza-y-desigualdad/otrosmetodos-de-pobreza

Instituto Nacional de Estadística y Censos de Costa Rica (2014). Estadísticas demográficas. 2011-2025. Proyecciones nacionales: Población total proyectada al 30 de junio por grupos de edades, según provincia, cantón, distrito y sexo [Fichero de datos]. Recuperado de http://www.inec.go.cr/poblacion/estimaciones-y-proyecciones-de-poblacion

Ministerio de Obras Públicas y Transportes (2013). Anuario De Información De Tránsito 2012. Recuperado de: http://repositorio. mopt.go.cr:8080/xmlui/bitstream/handle/123456789/399/388-4.pdf?sequence=1\&isAllowed=y

Pulugurthaa, S. S., Duddub, V. R. y Kotagiri, Y. (2013). Traffic analysis zone level crash estimation models based on land use. Accident Analysis and Prevention, 50, 678-687. DOI: 10.1016/j.aap.2012.06.016

Programa de Investigación en Desarrollo Urbano Sostenible (ProDUS) (2015). Costos de los choques viales en Costa Rica (Informe Final Proyecto 321-B0602). Recuperado de: https://www.ucr.ac.cr/medios/documentos/2015/informe-final.pdf

Wang, K., Ivan, J. N., Burnicki, A. C. y Mamun, S. A. (2017). Predicting local road crashes using socioeconomic and land cover data. Journal of Transportation Safety E Security, 9(3), 301-318.

World Health Organization (2018). Global status report on road safety 2018. Geneva: World Health Organization. 